\title{
Publisher Correction: Quantum mechanics: An inconsistent friend
}

Matthew F. Pusey

Correction to: Nature Physics https://doi.org/10.1038/s41567-018-0293-7, published online 18 September 2018.

In the version of this News \& Views originally published, the link for ref. 7 was incorrectly given as https://arxiv.org/abs/1604.07422; it should have been https://scirate.com/arxiv/1604.07422. This has now been corrected. 